\title{
Involvement of autophagy in diosgenin-induced megakaryocyte differentiation in human erythroleukemia cells
}

\author{
DIMA DIAB ${ }^{1}$, ALINE PINON $^{1}$, CATHERINE OUK $^{2}$, ROUBA HAGE-SLEIMAN $^{3}$, \\ MONA DIAB-ASSAF ${ }^{4}$, BERTRAND LIAGRE $^{1 *}$ and DAVID YANNICK LEGER ${ }^{1^{*}}$ \\ ${ }^{1}$ PEIRENE Laboratory EA 7500, Faculty of Pharmacy; ${ }^{2}$ BISCEm Flow Cytometry/Microscopy Unit, \\ University of Limoges, 87025 Limoges, France; ${ }^{3}$ Department of Biology, Faculty of Sciences; ${ }^{4}$ Doctoral School of \\ Sciences and Technology, Lebanese University, Hadath El Jebbeh, Beyrouth 21219, Lebanon
}

Received April 20, 2021; Accepted July 21, 2021

DOI: $10.3892 / \mathrm{mmr} .2021 .12386$

\begin{abstract}
Natural agents have been used to restart the process of differentiation that is inhibited during leukemic transformation of hematopoietic stem or progenitor cells. Autophagy is a housekeeping pathway that maintains cell homeostasis against stress by recycling macromolecules and organelles and plays an important role in cell differentiation. In the present study, an experimental model was established to investigate the involvement of autophagy in the megakaryocyte differentiation of human erythroleukemia (HEL) cells induced by diosgenin [also known as (25R)-Spirosten-5-en-3b-ol]. It was demonstrated that Atg7 expression was upregulated from day 1 of diosgenin-induced differentiation and was accompanied by a significant elevation in the conversion of light chain 3 A/B (LC3-A/B)-I to LC3-A/B-II. Autophagy was modulated before or after the induction of megakaryocyte differentiation using 3-methyladenine (3-MA, autophagy inhibitor) and metformin (Met, autophagy initiation activator). 3-MA induced a significant accumulation of the LC3 A/B-II form at day 8 of differentiation. It was revealed that 3-MA had a significant repressive effect on the nuclear (polyploidization) and membrane glycoprotein $\mathrm{V}[(\mathrm{GpV})$ expression] maturation.
\end{abstract}

Correspondence to: Professor Bertrand Liagre, PEIRENE Laboratory EA 7500, Faculty of Pharmacy, University of Limoges, 2 Rue du Docteur Raymond Marcland, 87025 Limoges, France

E-mail: bertrand.liagre@unilim.fr

${ }^{*}$ Contributed equally

Abbreviations: ELISA, enzyme-linked immunosorbent assay; FACS, fluorescence-activated cell sorter; FBS, fetal bovine serum; GpV, glycoprotein V; HEL, human erythroleukemia; HRP, horseradish peroxidase; LC3A/B, light chain $3 \mathrm{~A} / \mathrm{B}$; 3-MA, 3-methyladenine; Met, metformin; MK, megakaryocytes; PBS, phosphate buffered saline; PI, propidium iodide; PVDF, polyvinylidene fluoride

Key words: diosgenin, human erythroleukemia cells, megakaryocyte differentiation, autophagy, 3-methyladenine
On the other hand, autophagy activation increased $\mathrm{GpV}$ genomic expression, but did not change the nuclear maturation profile after HEL cells treatment with Met. It was concluded that autophagy inhibition had a more prominent effect on the diosgenin-differentiated cells than autophagy activation.

\section{Introduction}

The differentiation therapy of cancer cells is an alternative therapeutic strategy used to treat leukemia regarding its lower side effects in comparison with the traditional chemotherapy $(1,2)$. Natural agents have been used to restart the process of differentiation which was stopped during leukemic transformation of hematopoietic stem cells or progenitor (3-5). In this context, previously in our laboratory, Beneytout et al demonstrated that treating human erythroleukemia (HEL) cells with $10 \mu \mathrm{M}$ of a natural steroidal saponin named diosgenin [(25R)-5-spirosten-3b-ol] induced its megakaryocytic differentiation (6). Formerly, published results showed that diosgenin-induced HEL megakaryocytic differentiation is accomplished after 8 days. With time progression, the main features of the megakaryocytic maturation began to stand out as progressive cell enlargement and polyploidization e.g. the increase in the DNA content up to 64N. Some cell receptors expression as CD41, a marker of the megakaryocytic differentiation, appeared and increased continuously until the end of the differentiation with the regression of the erythroid marker glycophorin A $(7,8)$. Many teams in which our laboratory confirmed that the platelet formation from mature megakaryocytes implicates apoptotic signaling pathways and key effectors activation (9-11). However, other researchers reported that the caspase-3, poly-ADP ribose polymerase (PARP) cleavage are not only correlated to the megakaryocyte's fragmentation driven by apoptosis $(12,13)$. In 2006, Leger et al demonstrated that, during the initial differentiation stages, diosgenin-treated HEL cells undergo a transient apoptosis-independent caspase-3 activation that could be necessary to start the demarcation membrane system development (9).

Autophagy is a housekeeping pathway that maintains cell homeostasis against stress by recycling macromolecules and organelles and plays an important role in cell development, death, survival, and differentiation. There are different 
types of autophagy: macroautophagy, microautophagy, and chaperone-mediated autophagy. We were interested in macroautophagy in which cytoplasmic materials are going to be enclosed in a double membrane structure called autophagosomes to be recycled and degraded. This autophagy canonical pathway is composed of three parts: the initiation, the elongation, and the autophagosome maturation $(14,15)$. Autophagy is modulated by many chemical molecules, among them 3-methyladenine (3-MA) and metformin (Met). 3-MA is an autophagy inhibitor that will inhibit the PI3K complex III during autophagy. Wu et al had demonstrated that the 3-MA is not specific for the PI3K complex III, but it is a selective inhibitor for the PI3K complex family. The principle evidence on the autophagy flux blockage is the accumulation of the form LC3A/B-II (Light chain $3 \mathrm{~A} / \mathrm{B}-\mathrm{II}$ ) as evidence of the lysosomal degradation interruption (16). Furthermore, Met is an autophagy initiation activator that plays essentially on the AMPK/LKB1 axis, an activator of the complex ULK1/2 (autophagy initiation complex). On the other hand, Met can abrogate the mTORC1 pathway that mainly regulates the autophagy (17).

Interestingly, in the last few years, scientists started to investigate the role of this second programmed cell death pathway in megakaryocytic differentiation. In 2009, Colosetti et al showed that the knockdown of the autophagy key effectors, Beclin1, LC3 abrogated the K562 megakaryocytic differentiation induced by phorbol myristate acetate (18). Cao et al confirmed that the complete knockout Atg7 -/- mice abrogate the megakaryocytopoiesis and the thrombopoiesis (19). In 2017, Wang et al demonstrated that the autophagy inhibition or activation in fetal liver cells but not in mature megakaryocytes altered significantly the megakaryocytopoiesis and thrombopoiesis (20).

In our present study, we establish an experimental model to investigate the autophagy involvement in the HEL megakaryocytic differentiation induced by the diosgenin. We showed that the autophagy effector's protein expression was changed during the diosgenin-induced megakaryocytic differentiation. We proceed with our investigation by modulating autophagy before and after the differentiation induction to target the maximum of the critical point that affect this procedure. At a late stage of the differentiation, we showed that the autophagy inhibition by 3-MA had a significant repression effect on the nuclear (polyploidization) and membrane (Glycoprotein $\mathrm{V}$ $(\mathrm{GpV})$ expression) maturation. On the other hand, autophagy activation increased the $\mathrm{GpV}$ genomic expression but did not changed the nuclear maturation profile after HEL cells treatment with Met. Given together, this study demonstrates that autophagy was implicated in the HEL megakaryocytic differentiation induced by diosgenin.

\section{Materials and methods}

Materials. RPMI-1640 medium, fetal bovine serum (FBS), L-glutamine and penicillin-streptomycin were purchased from Gibco BRL. Diosgenin [(25R)-5-spirosten-3 $\beta$-ol], 3-methyladenine (3-MA), metformin (Met) were obtained from Sigma-Aldrich. The monoclonal antibody against Beclin1, Atg7, Atg12-5, Atg3, LC3A/B were acquired from Cell Signaling Technology-Ozyme (Saint-Cyr-L'école,
France). GAPDH antibody was purchased from Santa Cruz Biotechnology (Santa Cruz Biotechnology-Clinisciences (Nanterre, France). Goat anti-rabbit IgG H\&L horseradish peroxidase (HRP) secondary antibody was purchased from Abcam (Paris, France). Rabbit anti-mouse IgG-IgM H\&L HRP secondary antibody and propidium iodide (PI) were obtained from Invitrogen-Thermo Fisher Scientific, Inc. Immobilon Western Chemiluminescent HRP Substrate was acquired from Merck.

Cell line, cell culture and treatment. The HEL cell line was kindly provided by Professor J.P. Cartron (INSERM U76) (6). Cells were seeded at $10^{5}$ cells $/ \mathrm{ml}$ in tissue culture flasks, grown in RPMI-1640 medium (Gibco BRL) supplemented with $10 \%$ fetal calf serum (Gibco BRL), $1 \%$ sodium pyruvate, $1 \%$ HEPES (Gibco BRL), $100 \mathrm{U} / \mathrm{ml}$ penicillin and $100 \mu \mathrm{g} / \mathrm{ml}$ streptomycin. Cultures were maintained in a humified atmosphere with $5 \% \mathrm{CO}_{2}$ at $37^{\circ} \mathrm{C}$. Diosgenin is widely used in many pharmacological applications. Diosgenin is soluble in many organic solvents as propyl acetate, acetone, isopropanol, methanol and ethanol. As the literature suggest, diosgenin used on human cancer cells is dissolved in absolute ethanol. With the exception of ethanol at a final concentration of $0.1 \%$ in the culture medium, all the other solvents mentioned above cannot be used in cell culture. At 1995, in our laboratory, Beneytout et al confirmed that ethanol with a concentration below $0.1 \%$ do not induce any differentiation effect (6). In order to induce the megakaryocytic differentiation, the cells were treated with diosgenin $10 \mu \mathrm{M}$ after 1 day of seeding and left for 10 days. The same amount of vehicle $(<0.1 \%$ ethanol) was added to control cells. The autophagy modulation was done by 3-MA ( $2 \mathrm{mM})$ and Met $(0.25 \mathrm{mM})$. We pretreated the HEL cells with 3-MA or Met $2 \mathrm{~h}$ prior to the differentiation induction by diosgenin $(10 \mu \mathrm{M})$ or later at day 2 or day 4 of the differentiation. Then, the cells were harvested at day 1, 2, 4, 6 and 8 of the differentiation, washed twice in phosphate buffered saline (PBS, pH 7.4), counted and cell viability was determined by the trypan blue dye exclusion method. Before starting our experiment, we tried to see whether 3-MA and Met induce the megakaryocytic differentiation. There is no difference between the control group $(<0.1 \%$ ethanol) and the treated group with different concentrations during 24 and $48 \mathrm{~h}$ (data not shown). The photos do not reflect any morphological changes comparing to the control group.

RNA extraction and semi-quantitative RT-PCR analysis. Total RNA was isolated from HEL cells with the RNeasy ${ }^{\circledR}$ Mini Kit following the RNA extraction and semi-quantitative RT-PCR manufacturer's protocol (Qiagen). $2 \mu \mathrm{g}$ total RNA were retro-transcribed into cDNA using the first-strand cDNA synthesis part of Omniscript RT Kit (Qiagen), oligo-dT (25 $\mu \mathrm{g})$ (Invitrogen, Cergy Pontoise, France) and RNase Out ${ }^{\mathrm{TM}}$ (40 U/ml) (Invitrogen). $2 \mu \mathrm{l}$ of reverse-transcribed cDNA was used for PCR according to the Kit HotStar Taq DNA Polymerase (250 units) protocol (Qiagen) with dNTP mix (100 mM) (Invitrogen) and $0.5 \mu \mathrm{M}$ of sense and antisense primers. The primers for PCR were chosen to amplify human GpV and we used GAPDH as an internal control. PCR resulting fragments were visualized as previously described (21) by the GBOX (Syngene) and the 
Genesnap version 7.09 software. Bands quantification was done by Image $\mathbf{J}$ software.

Evaluation of nuclear ploidy. For DNA content analysis for all conditions, $10^{6}$ cells were fixed and permeabilized in $70 \%$ ethanol in PBS at $-20^{\circ} \mathrm{C}$ overnight, washed in PBS, treated with RNase (40 U/ $\mu 1$, Boehringer Mannheim) for $20 \mathrm{~min}$ at room temperature, and stained with PI $(50 \mu \mathrm{g} / \mathrm{ml})$. Flow cytometric analyses (fluorescence-activated cell sorter (FACS) were performed as described previously (22) (Becton Dickinson FACScalibur). The used software was CellQuest ${ }^{\mathrm{TM}}$ pro version 6.0 (Becton Dickinson).

Protein expression analysis. For total protein extraction, control or treated were washed in PBS, then, the total cell pool was centrifuged at $200 \mathrm{~g}$ for $5 \mathrm{~min}$ at $4^{\circ} \mathrm{C}$ and homogenized in RIPA lysis buffer (50 mM HEPES, pH 7.5, $150 \mathrm{mM} \mathrm{NaCl}$, $1 \%$ sodium deoxycholate, $1 \% \mathrm{NP}-40,0.1 \% \mathrm{SDS}, 20 \mathrm{mg} / \mathrm{ml}$ of aprotinin) containing protease inhibitors (Complete ${ }^{\mathrm{TM}} \mathrm{Mini}$, Roche Diagnostics) according to the manufacturer's instructions. Protein levels were determined using the Bradford method. Proteins (40-70 $\mu \mathrm{g})$ were separated by electrophoresis on $10-12 \%$ SDS-PAGE gels and transferred to polyvinylidene fluoride (PVDF) membranes (Amersham Pharmacia Biotech). Western blotting was performed on following proteins with respective antibodies against: Beclin1, Atg7, Atg12-5, Atg3, LC3A/B: (1:1,000). After incubation with the appropriate secondary antibodies, blots were developed using the Immobilon Western Chemiluminescent HRP Substrate and G:BOX system (Syngene) (23) and Genesys version 1.6.1.0 software. The bands density are measured by using Image $\mathbf{J}$ software. Membranes were then reblotted with human anti-GAPDH $(1: 1,000)$ used as a loading control.

DNA fragmentation. HEL cells were seeded at $10^{5}$ cells $/ \mathrm{ml}$ in $75 \mathrm{~cm}^{2}$ tissue culture flasks and then treated as described above. DNA fragmentation was quantified by 'cell death' enzyme-linked immunosorbent assay (ELISA) (Cell Death Detection ELISA $^{\text {PLUS }}$, Sigma-Aldrich, Saint-Quentin-Fallavier, France). Cytosol extracts were obtained from treated or control cells according to the manufacturer's protocol and DNA fragmentation was measured as previously described (24) by Thermo Scientific ${ }^{\mathrm{TM}}$ Multiskan ${ }^{\mathrm{TM}}$ FC Microplate Photometer (ThermoFisher SCIENTIFIC, Illkirch, France).

Statistical analysis. All quantitative results are expressed as the mean \pm standard error of the mean (SEM) of separate experiments using Excel (Microsoft Office, Version 98). Statistical significance was evaluated by the two-tailed unpaired Student's t-test. A P-value of $<0.05$ was considered to indicate significance.

\section{Results}

Autophagy flux is stimulated during diosgenin-induced megakaryocytic differentiation of HEL cells. We first wanted to study the autophagy protein expression during diosgenin-induced megakaryocytic differentiation of HEL cells. We studied the protein expression of different key autophagy mediators (Beclin1, Atg7, Atg12-5, Atg3, LC3A/B-I) and LC3A/B-II at days 1,2,4,6 and 8 of the differentiation (Fig. 1A). As the Fig. 1A indicates, in comparison with control cells, western blot results showed a significantly higher expression of Atg7 starting from day 1 . This pattern continued until the end of diosgenin-induced megakaryocytic differentiation of HEL cells (day 2, 4, 6 and 8, $\mathrm{P}<0.05)$. In parallel, we did not see any significant changes in the expression of Beclin1, Atg12-5 and Atg3 with the differentiation enhancement. Moreover, we noted a significant increase in the conversion of the form LC3A/B-I to LC3A/B-II at the last four days of the differentiation in comparison with control cells (day 4: 4.7-fold and day 8: 5.3-fold, $\mathrm{P}<0.05$ ) (Fig. 1B). This result showed that the autophagy flux was activated from day 1 after treating HEL with diosgenin and this activation was characterized by the continuously increased expression of autophagy initiation protein Atg7 and the conversion of the form LC3A/B-I to LC3A/B-II, an hallmark of autophagosome maturation.

Autophagy modulation by 3-MA but not Met affects the expression of autophagy mediators at the end of the diosgenin-induced megakaryocytic differentiation of $H E L$ cells. In order to define precisely the autophagy role during megakaryocytic differentiation, we modulated this pathway before and after the HEL cells treatment with diosgenin. The modulation was done by using two molecules that affect the autophagy initiation: Met (activator) and 3-MA (inhibitor). First, we decided to modulate autophagy $2 \mathrm{~h}$ before the differentiation induction since we wanted to see if autophagy has any effect on the differentiation beginning in a short term and the maturation in the long term. In the second place, we wanted to modulate autophagy in several time points after the differentiation launch: at day 2 (early stage of differentiation) and day 4 (cells reached a $32 \mathrm{~N}$ DNA content) (7). As the Fig. 1A illustrates, the autophagy inhibition by 3-MA before or after the HEL treatment with diosgenin inhibited the Atg7 protein expression but not Atg12-5 and Atg3. On days 2 and 4, Atg7 expression reduction was not statistically significant. On day 8, the protein expression of Atg7 was significantly diminished whether the autophagy was inhibited $2 \mathrm{~h}$ before the differentiation induction (2.5-fold) or after on day 4 (3.5-fold) in comparison with the cells treated only with diosgenin $(\mathrm{P}<0.05)$ (Fig. 1B). This abrogation was accompanied with an accumulation of the form LC3A/B-II $(\mathrm{P}<0.05)$ validating the autophagy flux repression (Fig. 1A and B). However, the autophagy activation by Met did not have any significant effect on the expression of Beclin1, Atg7, Atg12-5, Atg3 and the conversion of LC3A/B-I to LC3A/B-II independently from the treatment time (Fig. 1A and B). Taken together, the autophagy activation did not have any effect on the autophagy protein's expression whether at early or late-stage of the megakaryocytes differentiation. On the other hand, the autophagy inhibition by 3 -MA had an impact exclusively at a late stage (day 8 ) by inhibiting ATG7 expression and accumulating LC3-A/B-II.

Autophagy inhibition by 3-MA blocked polyploidization during diosgenin-induced megakaryocytic differentiation where autophagy induction did not affect the polyploidization process. Polyploidy is considered a major marker of the megakaryocytic differentiation by increasing the DNA content up to $128 \mathrm{~N}$ (25). Polyploidy was used by Léger et al (7) to evaluate the megakaryocytic differentiation 
A

\begin{tabular}{|c|c|c|c|c|c|c|}
\hline \multirow[b]{2}{*}{ Time (days) } & \multirow[b]{2}{*}{ Ctrl } & \multicolumn{5}{|c|}{ Dios $(10 \mu \mathrm{M})$} \\
\hline & & 1 & 2 & 4 & 6 & 8 \\
\hline \multicolumn{7}{|l|}{$2 \mathrm{~h}$ before } \\
\hline \multicolumn{7}{|l|}{2 days after } \\
\hline 4 days after & & & & & & \\
\hline
\end{tabular}

Beclin1

Atg7
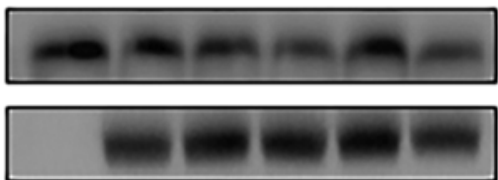

Atg12-5

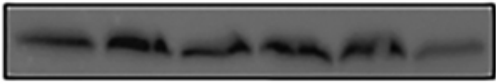

Atg3

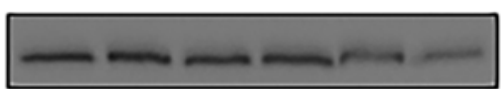

LC3A/B-I

LC3BA/B-II

GAPDH
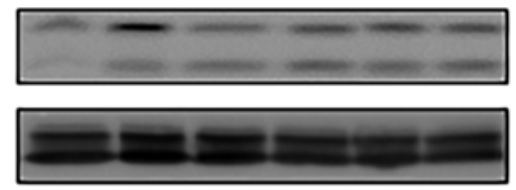
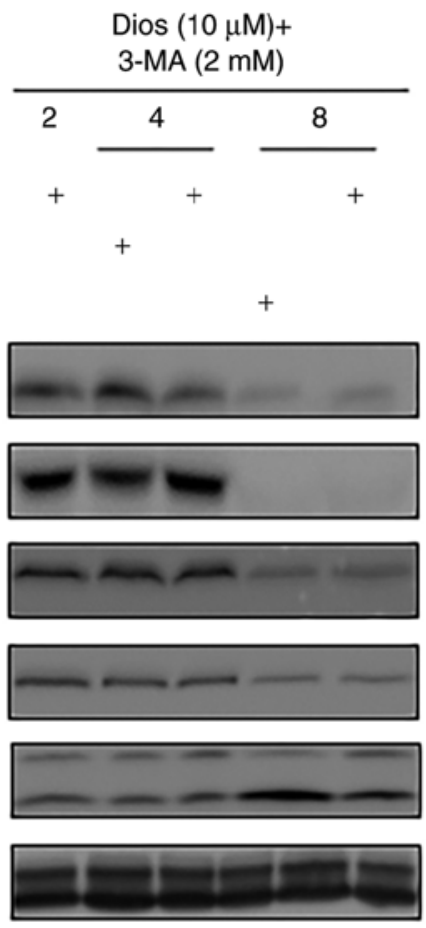

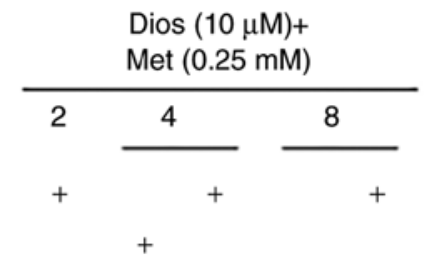

$+$
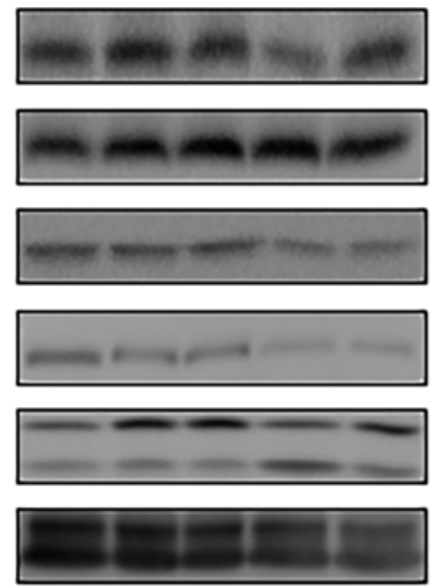

B

Atg7 口 LC3A/B-I

LC3A/B-II

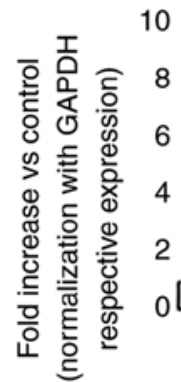

0
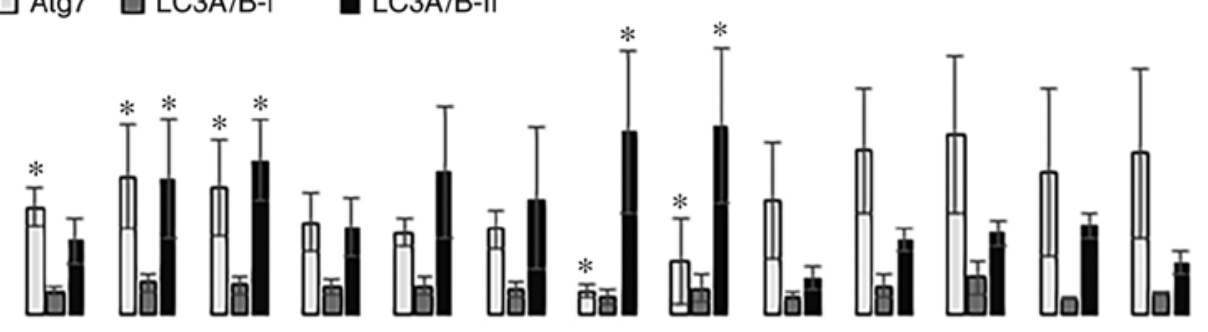

\begin{tabular}{|c|c|c|c|c|c|c|c|c|c|c|}
\hline \multirow[b]{2}{*}{ Time (days) } & \multirow[b]{2}{*}{ Ctrl } & \multicolumn{3}{|c|}{ Dios $(10 \mu \mathrm{M})$} & \multicolumn{3}{|c|}{$\begin{array}{c}\text { Dios }(10 \mu \mathrm{M})+ \\
\text { 3-MA }(2 \mathrm{mM})\end{array}$} & \multicolumn{3}{|c|}{$\begin{array}{l}\text { Dios }(10 \mu \mathrm{M})+ \\
\text { Met }(0.25 \mathrm{mM})\end{array}$} \\
\hline & & 2 & 4 & 8 & 2 & 4 & 8 & 2 & 4 & 8 \\
\hline $2 \mathrm{~h}$ before & & & & & + & + & + & + & + & + \\
\hline 2 days after & & & & & & & & & + & \\
\hline 4 days after & & & & & & & & & & \\
\hline
\end{tabular}

Figure 1. Time-dependent expression of autophagy-related protein expression during diosgenin-induced megakaryocytic differentiation in human erythroleukemia cells and effect of autophagy inhibitor or activator. (A) Beclin1, Atg7, Atg12-5, Atg3 and LC3A/B expression levels were evaluated in the total cellular pool using western blot analysis (GAPDH was used as a loading control and the blot shown is representative of three separate experiments). (B) The fold increase values are expressed as the mean \pm SEM $(n=3)$. "P<0.05 vs. ctrl group. Dios, diosgenin; LC3A/B, light chain 3 A/B; Ctrl, control; 3-MA, 3-methyladenine; Met, metformin.

of HEL cells after treatment with diosgenin. Polyploidy in the diosgenin-differentiated cells reach out $64 \mathrm{~N}$ at the end of the differentiation (day 8) (7). Based on this, we evaluated the polyploidization in the diosgenin-differentiated cells after autophagy modulation by 3-MA or Met. The results in Fig. 2 show that the autophagy inhibition by 3-MA reduced polyploidization at day 4 and 8 of the differentiation but not at an early stage (day 2). Moreover, on day 4 and 8 , we observed a strong inhibition of the polyploidy if the HEL cells were $2 \mathrm{~h}$ pre-treated by 3 -MA $(55 \%$ and $45 \%$ of inhibition respectively vs. the cells treated only with diosgenin on days 4 and 8). These inhibition rates were significantly higher in comparison with the cells in which the autophagy was inhibited after the differentiation induction (day 2 or day 4) by diosgenin $(33 \%$ and $22 \%$ of inhibition respectively vs. the cells treated only with diosgenin at day 4 and 8). On the contrary, independently from the treatment time, autophagy activation by Met did not had any impact on the polyploidization (Fig. 2). Collectively, these results demonstrated a strong autophagy inhibition effect on polyploidy on day 4 and 8 , which is not the case after autophagy activation.

Autophagy inhibition blocked GpV transcription where autophagy induction increased $G p V$ transcription during 
100

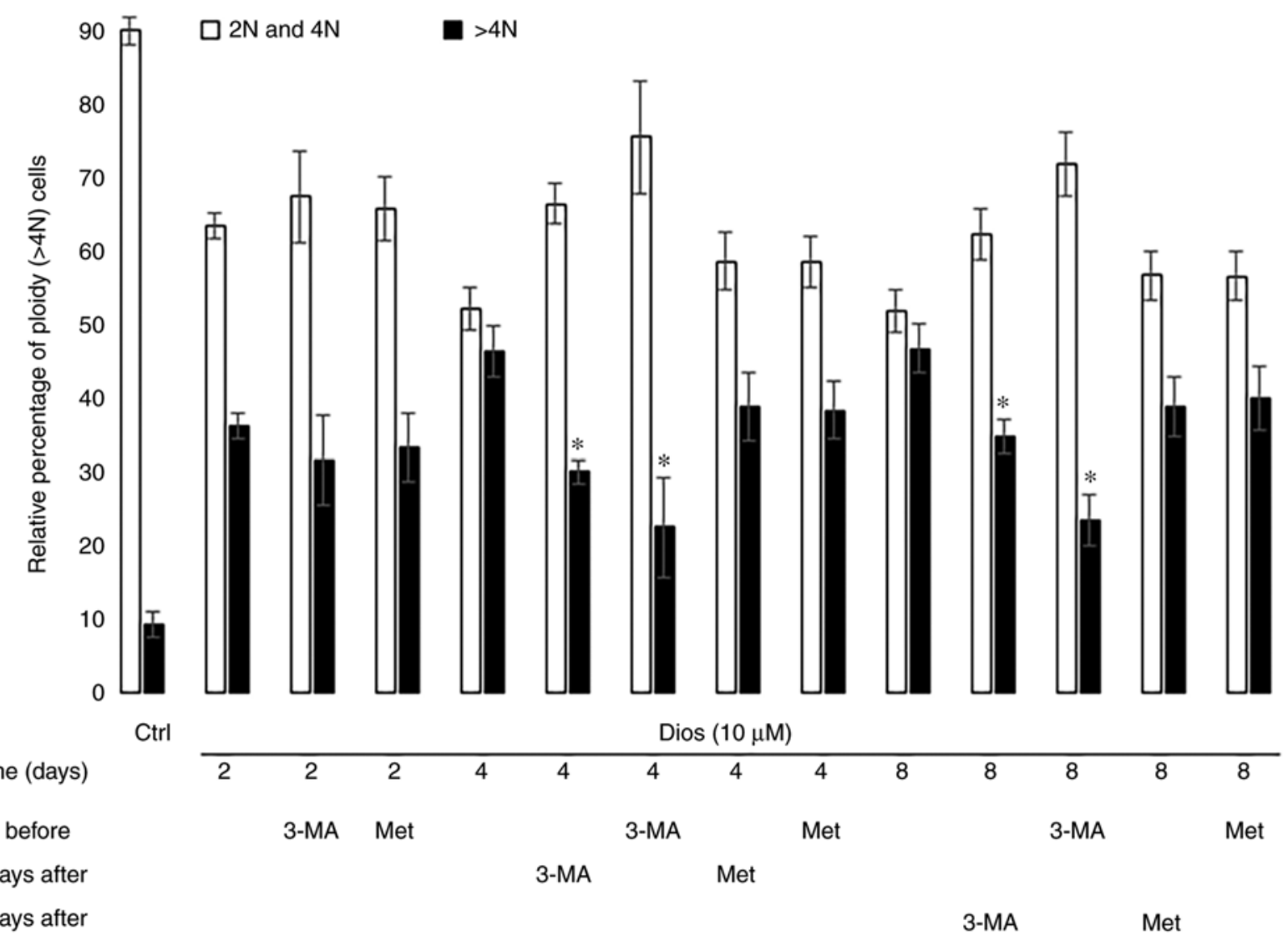

Figure 2. Effect of autophagy inhibitor or activator on cell ploidy during diosgenin-induced megakaryocyte differentiation in human erythroleukemia cells. Relative percentage of nuclear ploidy classes were determined by flow cytometry and values are expressed as the mean $\pm \mathrm{SEM}$ ( $\mathrm{n}=3$ ). ${ }^{*} \mathrm{P}<0.05$ vs. dios alone group (>4N). Dios, diosgenin; Ctrl, control; 3-MA, 3-methyladenine; Met, metformin.

diosgenin-induced megakaryocytic differentiation. The next step was to see the effect of the autophagy modulation on the megakaryocytes membrane receptor expression. GpV was used to identify the maturation level after the induction of HEL cells megakaryocytic differentiation by diosgenin (7). As the Fig. 3 demonstrates, 3-MA induced a dramatic decrease in $\mathrm{GpV}$ transcription during diosgenin-induced megakaryocytic differentiation (Fig. 3A). This decrease occurred after 4 days of diosgenin-induced differentiation, regardless of the treatment time at which 3-MA was added (2-fold 2 days after and 9-fold $2 \mathrm{~h}$ before, $\mathrm{P}<0.05)$ (Fig. 3B). This decrease was even more drastic $(>13$-fold, $\mathrm{P}<0.05)$ at the end of differentiation and was correlated with the decrease observed in polyploidization process after 3-MA treatment (Figs. 2 and 3).

Concerning the effect of autophagy induction, we observed that Met induced an increase in $\mathrm{GpV}$ transcription during diosgenin-induced megakaryocytic differentiation. This increase started after 4 days of diosgenin-induced differentiation and lasted until the end of the treatment (day 8) regardless of the treatment time at which Met was added (1.6-fold and 1.7-fold, $\mathrm{P}<0.05$ ) (Fig. 3A and B).

The autophagy flux modulation by 3-MA or Met does not affect DNA fragmentation of HEL cells. In 2002, De Botton et al reported that during the early stage of the megakaryocytic differentiation, caspase- 3 activity was detected but not related to any DNA fragmentation signal (12). Here, we investigated the effect of autophagy modulation during the megakaryocytic differentiation of HEL cells induced by diosgenin on DNA fragmentation. As the Fig. 4 demonstrates, the DNA fragmentation fold increase compared to control cells starting from day 2 to the end of the treatment. As the Fig. 4A and B report, the autophagy modulation $2 \mathrm{~h}$ before or on day 2 or day 4 after the HEL cells treatment with diosgenin did not significantly changed DNA fragmentation process.

\section{Discussion}

Most of the anti-cancer treatments aimed to induce cancer cell cytotoxicity. Recently, cancer cell differentiation is considered as an additional choice to eradicate cancer cells. Talking about leukemia, differentiation therapy had been studied by many teams. Several molecules are engaged in this context and many of them are natural compounds such as retinoic acid (26). In our laboratory, the diosgenin-induced megakaryocytic differentiation of HEL cells had been studied since 1995 by Beneytout et al (6). Léger et al investigated the molecular mechanisms induced after HEL cells treatment with diosgenin. According to these works, apoptosis is indispensable for mature megakaryocyte's fragmentation and pseudo-platelet release in vitro (7-9). Recently, the implication of the autophagy pathway during the megakaryocytic 
A
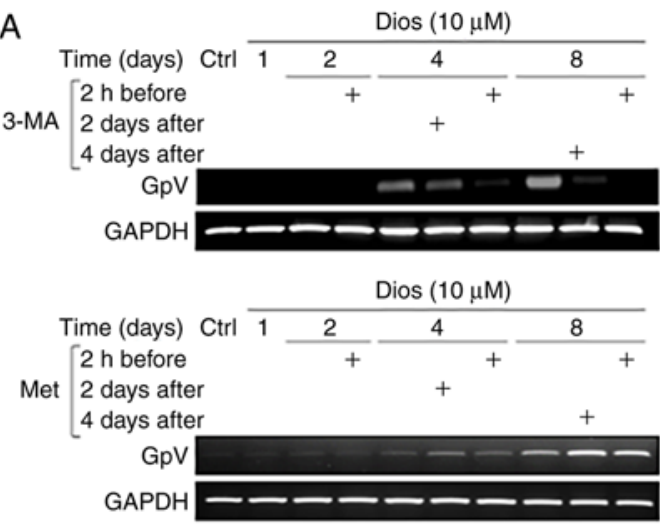

B
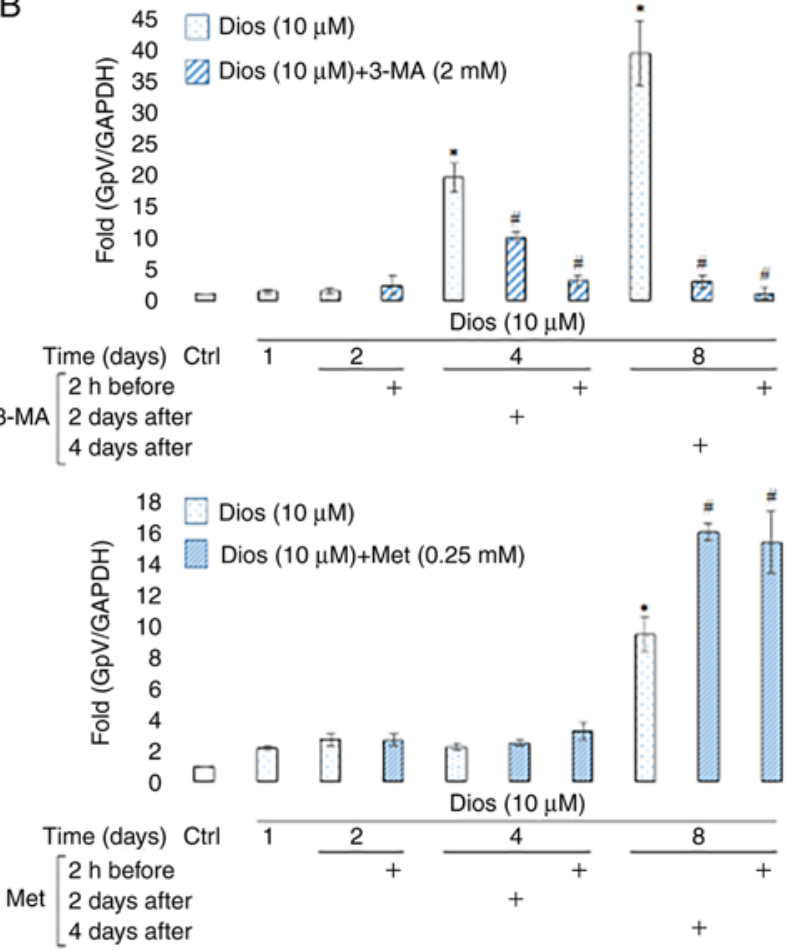

Figure 3. Effect of autophagy inhibitor or activator on $\mathrm{GpV}$ mRNA expression during diosgenin-induced megakaryocyte differentiation in human erythroleukemia cells. (A) Reverse transcription-semi-quantitative PCR analysis was performed after total RNA extraction and semi-quantification of $\mathrm{GpV}$ transcripts using GAPDH as an internal control. Agarose gels shown are representative of three separate experiments. (B) The fold ratios are expressed as the mean $\pm \operatorname{SEM}(\mathrm{n}=5)$. ${ }^{*} \mathrm{P}<0.05$ vs. ctrl group; ${ }^{*} \mathrm{P}<0.05$ vs. dios group. Dios, diosgenin; Ctrl, control; 3-MA, 3-methyladenine; Met, metformin; $\mathrm{GpV}$, glycoprotein V.

differentiation had been discussed by the scientific community $(18,19,27)$. This assumption was built on the existence of common regulatory pathways between those two procedures. A study conducted by Cao et al demonstrated that Atg7 knockout in mice impaired the megakaryocytic differentiation and altered the thrombopoiesis (19). During the same year, an additional study by Ouseph et al confirmed that the Atg7 knockdown in mature megakaryocytes does not have any effect on it (27). Autophagy's precise role during the megakaryocytic differentiation remain poorly understood and needs more investigations to be clarified.

Autophagy is a homeostatic pathway aiming to recycle the cytosolic content in cells (14). Since 1970, many pathologies linked to megakaryocytes as ITP (immune thrombocytopenia) describe the occurrence of autophagy vacuoles in those cells (28). We are interested in macro-autophagy. This non-selective process implicates several Atgs that will make two ubiquitin-like conjugation systems consisted of Atg7, Atg10, Atg12, Atg5, Atg16L1 and Atg3. Atgs will conjugate LC3A/B-I to phosphatidylethanolamine on the autophagosome membrane and induce its enclosure (14). Our first step was to see the key autophagy mediator's protein expressions. We demonstrated that Atg7 expression is overexpressed since day 1 after the differentiation induction by diosgenin and was accompanied by a significant elevation of the LC3-A/B-I conversion to LC3-A/B-II. This first result demonstrates that the autophagy flux is active during the diosgenin-induced megakaryocytic differentiation of HEL cells. Given that the Atg3 and Atg12-5 protein expressions were unchanged during the diosgenin-induced megakaryocytic differentiation, the autophagy non-canonical pathway (a pathway independent from Beclin1, Atg12-5 and Atg3) could be activated but this hypothesis remains under investigation. A published study in 2009 by Colosetti et al mentioned that the autophagy genes (Beclin1 and LC3) knockdown in K562 cells impeded the induced megakaryocytic differentiation marker CD41 ${ }^{+}$ (Glycoprotein II-B) (18). Moreover, a study conducted by Huang et al in 2011 reported that the autophagy inhibition by 3 -MA altered the lapatinib-induced megakaryocytic differentiation of K562 cells (29). Recently, a published article by Wang et al confirmed previous studies by demonstrating that the autophagy modulation by bafilomycin A1 (autophagy inhibitor) and rapamycin (mTORC1 inhibitor) in fetal liver cells abrogated the polyploidy as well as the expression of the principle megakaryocytic maturation markers (20). However, autophagy modulation in mature megakaryocytes does not affect these parameters (20). In our present study, we modulated autophagy before or after the megakaryocytic differentiation induction by using Met and 3-MA. We have mentioned that Met is never been used in the megakaryocytic differentiation study field. We noted that Met did not have any significant effect on the autophagy flux in the diosgenin-differentiated cells independently from the treatment strategy. This is normal to be reflected on the $\mathrm{GpV}$ expression. There is no difference between the polyploidy and the $\mathrm{GpV}$ genomic expression after autophagy activation at the second and the fourth day of differentiation. Thus, the autophagy activation by Met in our study did not induce a statistically significant accumulation of the LC3A/B-II in HEL cells, indicating the necessity to use another activator agent to decide if this is the case in our cell line. Further, to explain the Met behavior in our case, additional investigations should be done regarding the detection of AMPK/LKB1 axis and phospho-Akt status.

However, 3-MA induced a significant accumulation of the form LC3 A/B-II at day 8 of the differentiation and not before. It is important to note that 3-MA is a selective PI3K complex family inhibitor (16) therefore, more investigations should be done regarding the $\mathrm{PI} 3 \mathrm{~K} / \mathrm{mTORC1}$ /Akt axis since mTORC1 is essential for the megakaryocytic differentiation (30). In order to see the effect of these observations on the megakaryocytic differentiation, we examined the nuclear maturation (polyploidy) of the diosgenin-differentiated megakaryocytes. In mature megakaryocytes, the polyploidy 


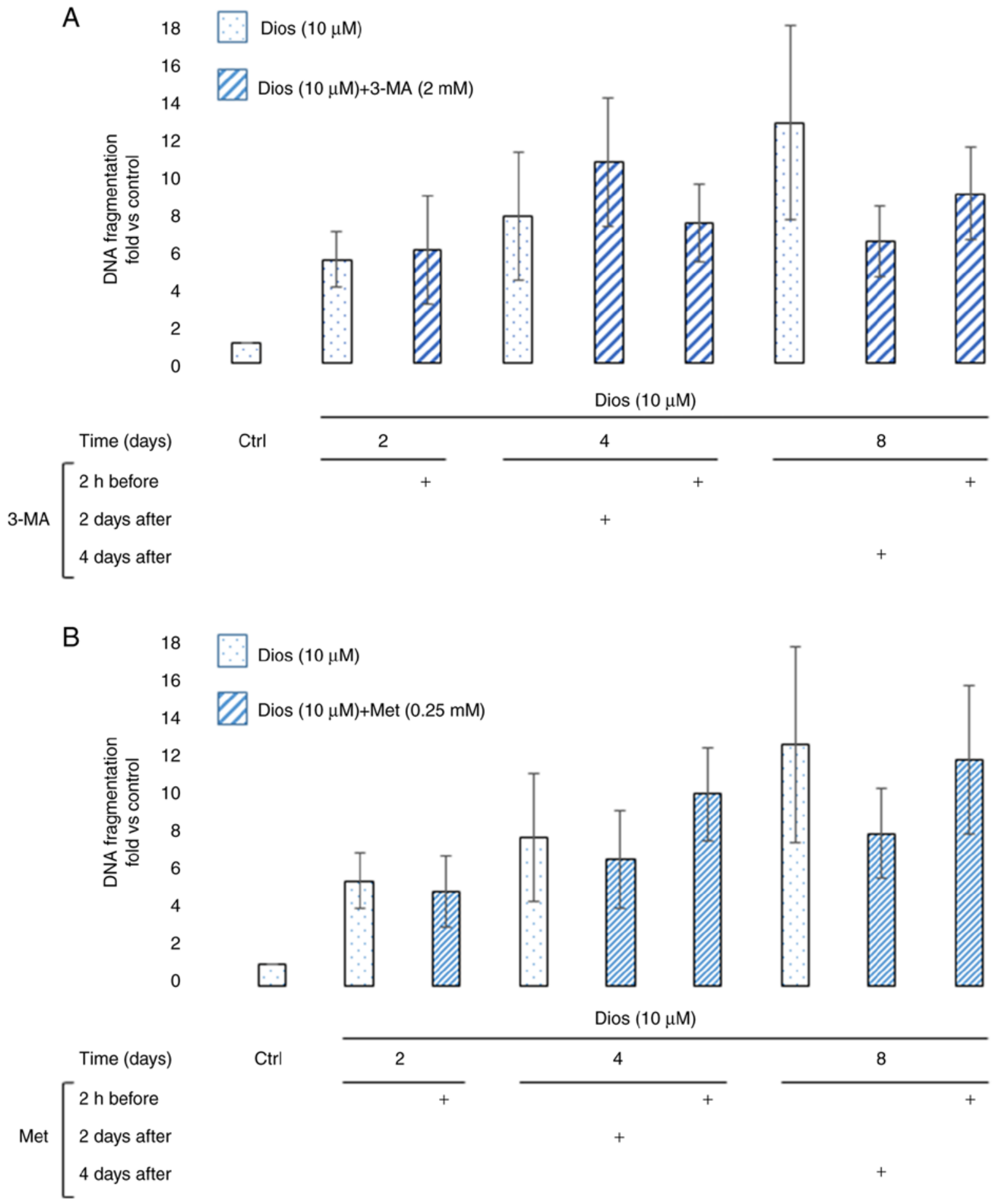

Figure 4. Effect of autophagy (A) inhibitor or (B) activator on DNA fragmentation during diosgenin-induced megakaryocytic differentiation in human erythroleukemia cells. DNA fragmentation was quantified from cytosol extracts by ELISA. Results are reported as n-fold compared with the control. Data are shown as the mean \pm SEM ( $\mathrm{n}=3$ ). Dios, diosgenin; Ctrl, control; 3-MA, 3-methyladenine; Met, metformin.

rate could reach $128 \mathrm{~N}$ and its importance lies in the cell size enlargement and platelet formation (25). In 2006, Drayer et al reported that the autophagy activation by rapamycin in $\mathrm{MO} 7 \mathrm{e}$ cells and human megakaryocytic primary progenitors does not affect the megakaryocytic polyploidization after the treatment with thrombopoietin (31). Another published work in 2020 by Sun et al reports that the autophagy activation by rapamycin in TPA (12-O-Tetradecanoylphorbol 13-acetate)-differentiated megakaryocytes from Dami cells increased significantly the polyploidy in a dose-dependent manner (32). Nevertheless, the autophagy inhibition by bafilomycin A1 inhibits the polyploidy (32). In this paper, we demonstrated that megakaryocytes are insensitive to the autophagy modulation on day 2 as the polyploidy rate did not change after modulating autophagy $2 \mathrm{~h}$ before the differentiation induction. On days 4 and 8 , Met continues to show no effect on the polyploidization independently from the treatment time. Met did not affect the key autophagy mediator's protein expression so this is might 
be reflected by the absence of the effect on the polyploidy on days 2, 4 and 8. On the other side, 3-MA inhibited the nuclear maturation of the diosgenin-induced megakaryocytes at days 4 and 8 whatever is the treatment time. This effect is stronger if the autophagy is inhibited $2 \mathrm{~h}$ before the treatment of HEL cells with diosgenin, which is compatible with the above-mentioned studies. According to the literature, the autophagy depletion in the megakaryocytic progenitors impedes the megakaryocytic differentiation. According to Léger et al, after 5 min of diosgenin treatment, the cell signaling is modulated toward the differentiation (7). Our results suggested that the autophagy inhibition effect appears strongly at day 4 and not before. The role of autophagy is to ensure that the cells are in a good percentage of polyploidy. With a high polyploidy rate, the cells are able to produce all the necessary materials to launch the platelet formation. The role of autophagy in the platelet formation during the diosgenin induced megakaryocytic differentiation needs to be investigated as apoptosis is involved during this stage. According to Léger et al on day 2 of differentiation, a caspase- 3 activity peak is observed as a sign of the platelet formation initiation inside the differentiated megakaryocytes. Furthermore, they showed an increase in the cleaved PARP expression and a reduction in the phospho-ERK1/2 activity $(7,9)$.

The majority of the studies focus on the expression of $\mathrm{CD} 41^{+}$ and $\mathrm{CD}^{+} 1^{+}$to characterize the megakaryocytic differentiation. However, $\mathrm{GpV}$ is a specific marker of the megakaryocytic lineages described since 1994 (33). According to this publication, the $\mathrm{GpV}$ expression increase while the megakaryocytic differentiation progression. In 2006, Léger et al presented $\mathrm{GpV}$ as a marker of the diosgenin-induced megakaryocytic differentiation of HEL cells (8). According to this study, $\mathrm{GpV}$ will increase progressively with the differentiation enhancement (8). Here in our study, we measured the GpV genomic expression to evaluate the autophagy modulation effect on the megakaryocytic differentiation. As claimed by Sun et al, the activation of the autophagy flux by rapamycin in TPA-differentiated megakaryocytes increased the $\%$ of CD $41^{+}$ megakaryocytes (MK) (32). Liu et al in 2011 demonstrated that neonatal MKs (micro MKs) can achieve full maturation and a mature membrane receptor profile with a low polyploidy level (4N) (34). In our report, we demonstrated that the autophagy activation by Met before and after the megakaryocytic differentiation induce the genomic expression of $\mathrm{GpV}$ despite of the ineffectiveness of the autophagy activation on the diosgenin-differentiated cell's polyploidy and independently from the treatment strategy. It is interesting to note that autophagy inhibition with 3-MA reduced both membrane maturation $(\mathrm{GpV})$ and polyploidization and that autophagy induction with Met promoted only membrane maturation $(\mathrm{GpV})$ and not polyploidization.

In 2000, Falcieri et al demonstrated that megakaryocytes from late stages of differentiation did not show any signal of DNA fragmentation (35). Using electrophoresis analysis, they suggested that DNA fragmentation was not internucleosomal like the majority of the hematopoietic cell lines that undergo apoptosis thus the DNA fragments did not appear on the agarose gel (35). In 2002, De Botton et al reported an early caspase-3 activity localized in the cytosol and independent from apoptosis as it did not lead to DNA fragmentation (12).
Otherwise, in later stages of the megakaryocytic differentiation, the DNA fragmentation increased with diffused caspase-3 inside the cells (12). In 2018, a TUNEL assay done on histological cut belonged to immune thrombocytopenia patients demonstrated the absence of DNA fragmentation signals in fully mature megakaryocytes (36). Here in this report, the DNA fragmentation assay showed no statistical significant variation of the DNA fragmentation in the cells treated only with diosgenin or the cells that underwent autophagy modulation.

In conclusion, this study demonstrated for the first time the autophagy implication in the diosgenin-induced megakaryocytic differentiation of HEL cells. The autophagy implication during the megakaryocytic differentiation was studied after the modulation of this pathway by 3-MA and Met. We conclude that the autophagy modulation induced an effect on the diosgenin-induced megakaryocytic differentiation. It is interesting to note that autophagy inhibition with 3-MA reduced both membrane maturation and polyploidization and that autophagy induction with Met promoted only membrane maturation and not polyploidization.

\section{Acknowledgements}

The authors would like to acknowledge Dr Ludovic Bretin and Miss Lucie Paulus (University of Limoges, Limoges, France) for their technical assistance during the experiments. The authors would also like to thank Dr Jeanne Cook-Moreau (University of Limoges, Limoges, France) for their help with manuscript editing.

\section{Funding}

The present study was funded in part by the Ministry of Higher Education, Research and Innovation by the New Aquitaine Regional Council and by the Lebanese University of Beirut (Beirut, Lebanon).

\section{Availability of data and materials}

The datasets used and/or analyzed during the current study are available from the corresponding author on reasonable request.

\section{Authors' contributions}

DYL participated in research design. DD, AP and CO conducted the experiments. DD, BL and DYL performed data analysis. DD, BL and DYL wrote or contributed to the writing of the manuscript. RHS and MDA obtained doctoral funding and contributed to the design of the research. DYL and BL confirm the authenticity of all the raw data. All authors have read and approved the final manuscript.

\section{Ethics approval and consent to participate}

Not applicable.

\section{Patient consent for publication}

Not applicable. 


\section{Competing interests}

The authors declare that they have no competing interests.

\section{References}

1. Sell S: Leukemia: Stem cells, maturation arrest, and differentiation therapy. Stem Cell Rev 1: 197-205, 2005.

2. Nowak D, Stewart D and Koeffler HP: Differentiation therapy of leukemia: 3 decades of development. Blood 113: 3655-3665, 2009.

3. Gocek E and Marcinkowska E: Differentiation therapy of acute myeloid leukemia. Cancers (Basel) 3: 2402-2420, 2011.

4. Yang J, Qiu J, Hu Y, Zhang Y, Chen L, Long Q, Chen J, Song J, Rao Q, Li Y, et al: A natural small molecule induces megakaryocytic differentiation and suppresses leukemogenesis through activation of $\mathrm{PKC} \delta / \mathrm{ERK} 1 / 2$ signaling pathway in erythroleukemia cells. Biomed Pharmacother 118: 109265, 2019.

5. Gao X, Wu J, Zou W and Dai Y: Two ellagic acids isolated from roots of Sanguisorba officinalis L. promote hematopoietic progenitor cell proliferation and megakaryocyte differentiation. Molecules 19: 5448-5458, 2014.

6. Beneytout JL, Nappez C, Leboutet MJ and Malinvaud G: A plant steroid, diosgenin, a new megakaryocytic differentiation inducer of HEL cells. Biochem Biophys Res Commun 207: 398-404 1995.

7. Léger D, Battu S, Liagre B, Cardot P and Beneytout JL: Sedimentation field flow fractionation to study human erythroleukemia cell megakaryocytic differentiation after short period diosgenin induction. J Chromatogr A 1157: 309-320, 2007.

8. Léger DY, Battu S, Liagre B, Beneytout JL and Cardot PJP: Megakaryocyte cell sorting from diosgenin-differentiated human erythroleukemia cells by sedimentation field-flow fractionation Anal Biochem 355: 19-28, 2006.

9. Leger D, Liagre B and Beneytout JL: Role of MAPKs and NF-kappaB in diosgenin-induced megakaryocytic differentiation and subsequent apoptosis in HEL cells. Int J Oncol 28: 201-207, 2006.

10. Luff SA, Kao CY and Papoutsakis ET: Role of p53 and transcription-independent $\mathrm{p} 53$-induced apoptosis in shear-stimulated megakaryocytic maturation, particle generation, and platelet biogenesis. PLoS One 13: e0203991, 2018.

11. Kovuru N, Raghuwanshi S, Sharma DS, Dahariya S, Pallepati A and Gutti RK: Endoplasmic reticulum stress induced apoptosis and caspase activation is mediated through mitochondria during megakaryocyte differentiation. Mitochondrion 50: 115-120, 2020.

12. De Botton S, Sabri S, Daugas E, Zermati Y, Guidotti JE, Hermine O, Kroemer G, Vainchenker W and Debili N: Platelet formation is the consequence of caspase activation within megakaryocytes. Blood 100: 1310-1317, 2002.

13. Solier S, Fontenay M, Vainchenker W, Droin N and Solary E: Non-apoptotic functions of caspases in myeloid cell differentiation. Cell Death Differ 24: 1337-1347, 2017.

14. Glick D, Barth S and Macleod KF: Autophagy: Cellular and molecular mechanisms. J Pathol 221: 3-12, 2010.

15. Wesselborg S and Stork B: Autophagy signal transduction by ATG proteins: From hierarchies to networks. Cell Mol Life Sci 72: 4721-4757, 2015.

16. Wu YT, Tan HL, Shui G, Bauvy C, Huang Q, Wenk MR, Ong CN, Codogno P and Shen HM: Dual role of 3-methyladenine in modulation of autophagy via different temporal patterns of inhibition on class I and III phosphoinositide 3-kinase. J Biol Chem 285: 10850-10861, 2010.

17. Hawley SA, Gadalla AE, Olsen GS and Hardie DG: The antidiabetic drug metformin activates the AMP-activated protein kinase cascade via an adenine nucleotide-independent mechanism. Diabetes 51: 2420-2425, 2002.

18. Colosetti P, Puissant A, Robert G, Luciano F, Jacquel A, Gounon P, Cassuto JP and Auberger P: Autophagy is an important event for megakaryocytic differentiation of the chronic myelogenous leukemia K562 cell line. Autophagy 5: 1092-1098, 2009.
19. Cao Y, Cai J, Li X, Fang Y, Zhang S, Yuan N, Mao X and Wang J: Loss of autophagy leads to megakaryocytes differentiation failure and defective platelets function. Blood 124: 4148, 2014.

20. Wang Q, You T, Fan H, Wang Y, Chu T, Poncz M and Zhu L: Rapamycin and bafilomycin A1 alter autophagy and megakaryopoiesis. Platelets 28: 82-89, 2017.

21. Pinon A, Limami Y, Micallef L, Cook-Moreau J, Liagre B, Delage C, Duval RE and Simon A: A novel form of melanoma apoptosis resistance: Melanogenesis up-regulation in apoptotic B16-F0 cells delays ursolic acid-triggered cell death. Exp Cell Res 317: 1669-1676, 2011.

22. Léger D, Liagre B, Cardot P, Beneytout JL and Battu S: Diosgenin dose-dependent apoptosis and differentiation induction in human erythroleukemia cell line and sedimentation field-flow fractionation monitoring. Anal Biochem 335: 267-278, 2004.

23. Bretin L, Pinon A, Bouramtane S, Ouk C, Richard L, Perrin ML, Chaunavel A, Carrion C, Bregier F, Sol V, et al: Photodynamic therapy activity of new porphyrin-xylan-coated silica nanoparticles in human colorectal cancer. Cancers (Basel) 11: 1474, 2019.

24. Bertrand J, Liagre B, Ghezali L, Beneytout JL and Leger DY: Cyclooxygenase- 2 positively regulates Akt signalling and enhances survival of erythroleukemia cells exposed to anticancer agents. Apoptosis 18: 836-850, 2013.

25. Mazzi S, Lordier L, Debili N, Raslova H and Vainchenker W: Megakaryocyte and polyploidization. Exp Hematol 57: 1-13, 2018.

26. Klobuch S, Steinberg T, Bruni E, Mirbeth C, Heilmeier B, Ghibelli L, Herr W, Reichle A and Thomas S: Biomodulatory treatment with azacitidine, all-trans retinoic acid and pioglitazone induces differentiation of primary AML blasts into neutrophil like cells capable of ROS production and phagocytosis. Front Pharmacol 9: 1380, 2018.

27. Ouseph MM, Huang Y, Banerjee M, Joshi S, MacDonald L, Zhong Y, Liu H, Li X, Xiang B, Zhang G, et al: Autophagy is induced upon platelet activation and is essential for hemostasis and thrombosis. Blood 126: 1224-1233, 2015.

28. Ortega Aramburu JJ: Idiopathic thrombocytopenic purpura-autoimmune thrombocytopenia in children. An Esp Pediatr 23: 145-151, 1985 (In Spanish).

29. Huang HL, Chen YC, Huang YC, Yang KC, Pan HY, Shih SP and Chen YJ: Lapatinib induces autophagy, apoptosis and megakaryocytic differentiation in chronic myelogenous leukemia K562 cells. PLoS One 6: e29014, 2011.

30. Raslova H, Baccini V, Loussaief L, Comba B, Larghero J, Debili N and Vainchenker W: Mammalian target of rapamycin (mTOR) regulates both proliferation of megakaryocyte progenitors and late stages of megakaryocyte differentiation. Blood 107: 2303-2310, 2006.

31. Drayer AL, Olthof SG and Vellenga E: Mammalian target of rapamycin is required for thrombopoietin-induced proliferation of megakaryocyte progenitors. Stem Cells 24: 105-114, 2006.

32. Sun RJ, Yuan D, Liu SY, Zhu JJ and Shan NN: Rapamycin induces megakaryocytic differentiation through increasing autophagy in Dami cells. Blood Coagul Fibrinolysis 31: 310-316, 2020.

33. Takafuta T, Fujimura K, Kawano H, Noda M, Fujimoto T, Oda K, Shimomura T and Kuramoto A: Expression of platelet membrane glycoprotein V in human megakaryocytes and megakaryocytic cell lines: A study using a novel monoclonal antibody against GPV. Thromb Haemost 72: 762-769, 1994.

34. Liu ZJ, Italiano J Jr, Ferrer-Marin F, Gutti R, Bailey M, Poterjoy B, Rimsza L and Sola-Visner M: Developmental differences in megakaryocytopoiesis are associated with up-regulated TPO signaling through mTOR and elevated GATA-1 levels in neonatal megakaryocytes. Blood 117: 4106-4117, 2011.

35. Falcieri E, Bassini A, Pierpaoli S, Luchetti F, Zamai L, Vitale M, Guidotti L and Zauli G: Ultrastructural characterization of maturation, platelet release, and senescence of human cultured megakaryocytes. Anat Rec 258: 90-99, 2000.

36. Vrbensky JR, Nazy I, Toltl LJ, Ross C, Ivetic N, Smith JW, Kelton JG and Arnold DM: Megakaryocyte apoptosis in immune thrombocytopenia. Platelets 29: 729-732, 2018.

This work is licensed under a Creative Commons Attribution-NonCommercial-NoDerivatives 4.0 International (CC BY-NC-ND 4.0) License. 